OPEN ACCESS

Edited by:

Marcus Fraga Vieira, Universidade Federal de Goiás, Brazil

Reviewed by:

Fu-Lien Wu

University of Illinois at UrbanaChampaign, United States

Rafael Reimann Baptista, Pontifical Catholic University of Rio

Grande do Sul, Brazil

*Correspondence:

Zhi-Jie Zhang

sportspt@163.com

Specialty section: This article was submitted to

Biomechanics,

a section of the journal Frontiers in Bioengineering and

Biotechnology

Received: 23 September 2021 Accepted: 13 December 2021 Published: 10 February 2022

Citation:

Chang T-T, Zhu Y-C, Li Z, Li F, Li Y-P, Guo J-Y, Wang $X-Q$ and Zhang $Z-J$ (2022) Modulation in the Stiffness of Specific Muscles of the Quadriceps in Patients With Knee Osteoarthritis and Their Relationship With Functional Ability.

Front. Bioeng. Biotechnol. 9:781672. doi: 10.3389/fbioe.2021.781672

\section{Modulation in the Stiffness of Specific Muscles of the Quadriceps in Patients With Knee Osteoarthritis and Their Relationship With Functional Ability}

\author{
Tian-Tian Chang ${ }^{1}$, Yuan-Chun $Z h u^{2}$, Zhe $L i^{3}$, Feng $L i^{1}$, Ya-Peng $L i^{1}$, Jia-Yi Guo ${ }^{1}$, \\ Xue-Qiang Wang ${ }^{2,4}$ and Zhi-Jie Zhang ${ }^{1 *}$
}

${ }^{1}$ Rehabilitation Therapy Center, Luoyang Orthopedic Hospital of Henan Province, Orthopedic Hospital of Henan Province, Luoyang, China, ${ }^{2}$ Department of Sport Rehabilitation, Shanghai University of Sport, Shanghai, China, ${ }^{3}$ The First Clinical Medical School, Shaanxi University of Chinese Medicine, Xi'an, China, ${ }^{4}$ Department of Rehabilitation Medicine, Shanghai Shangti Orthopaedic Hospital, Shanghai, China

Deficits in the flexibility of the quadriceps are one of the risk factors for developing knee joint disorders. No studies have investigated the changes in the stiffness of the quadriceps muscle among patients with knee osteoarthritis (OA). Therefore, the purpose of this study was to investigate changes in the stiffness of specific-muscle of the quadriceps in patients with knee OA and their relationship with functional ability. Twenty-five patients with knee OA and 25 healthy, asymptomatic subjects were recruited in this study. The stiffness of the vastus lateralis $(\mathrm{VL})$, vastus medialis $(\mathrm{VM})$ and rectus femoris (RF) in all participants was evaluated using MyotonPRO at $60^{\circ}$ and $90^{\circ}$ flexion of the knee joint. The results of this study showed a greater VL stiffness in patients with knee OA than in healthy subjects at both $60^{\circ}$ and $90^{\circ}$ of knee flexion $(p<0.05)$. Significant differences in VL, VM and RF stiffness were obtained at different knee joint angles in individuals with and without knee OA $(p<0.05)$. In addition, there was a positive correlation between VL stiffness and the Western Ontario and McMaster Universities Osteoarthritis Index (WOMAC) scores in patients with Knee $\mathrm{OA}\left(60^{\circ}\right.$ of knee flexion: $r=0.508, p=0.010$; $90^{\circ}$ of knee flexion: $r=0.456, p=0.022$ ). These results indicate that there is an increase in VL stiffness in patients with knee OA compared with healthy, asymptomatic subjects, and the quadriceps stiffness was increased with knee flexion in both healthy subjects and patients with knee OA. VL stiffness is associated with WOMAC scores in patients with knee OA.

Keywords: stiffness, quadriceps, osteoarthritis, knee, vastus lateralis, WOMAC

\section{INTRODUCTION}

Osteoarthritis (OA) is the most common joint disorder around the world and it can affect many joints but most frequently occurs in the knee (Du et al., 2019). Knee osteoarthritis, characterized by knee joint degeneration, usually occurs during middle to older age (Michael et al., 2010). It is estimated that approximately $37 \%$ of individuals (aged $>45$ years) are affected by knee OA (Dillon et al., 2006). Furthermore, the prevalence of knee OA has increased because of the increasingly older and obese population worldwide (Hunter and Bierma-Zeinstra, 2019). As a result, knee OA results in a significant social and economic burden (Liu Q et al., 2018). The costs of medical care, indirect costs 
due to work loss and premature retirement caused by knee OA are escalating (Cross et al., 2014; Safiri et al., 2020). There are many risk factors for knee OA, including a high body mass index (BMI), quadriceps weakness, frequent kneeling and squatting, and participation in sports (Simic et al., 2021; Szilagyi et al., 2021).

The quadriceps muscle plays an important role in shock absorption and knee joint stability during walking and running (Davis et al., 2019; Ebihara et al., 2020). The reduced flexibility of the quadriceps may be one of the risk factors for the development of anterior knee pain, including patellofemoral pain syndrome and patellar tendinopathy, and tight quadriceps muscles may contribute to increasing knee joint stress during functional activities (Witvrouw et al., 2000; Witvrouw et al., 2001; Piva et al., 2005). Previous studies have used the knee range of motion to reflect the flexibility of the quadriceps in patients with knee OA (Cho et al., 2015; Lu et al., 2018; Perlman et al., 2019). However, this method is used to quantify the flexibility of groups of muscles, not individual muscles. The joint range of motion is influenced not only by skeletal muscle but also by noncontractile structures such as skin, tendons and the joint capsule (Geertsen et al., 2015; Andrade et al., 2016; Nordez et al., 2017). Thus, the method used to quantify the joint range of motion could not provide insight into changes in the stiffness of individual muscles. Furthermore, several studies have demonstrated that the stiffness of each individual muscle may be influenced by various factors, such as contraction (Avrillon et al., 2018), stretching (Hirata et al., 2016) and pathology (Zhang et al., 2014; Zhou et al., 2020). In recent years, some studies compared the difference in the stiffness of soft tissues between healthy subjects and patients with musculoskeletal disorders (such as low back pain, neck pain, and patellar tendinopathy) and explored the mechanical properties of soft tissue in these patients (Ishikawa et al., 2017; Breda et al., 2020; Koppenhaver et al., 2020). One study has investigated quadriceps tendon stiffness in patients with knee OA and they found the passive stiffness of the quadriceps tendon was negatively correlated to the maximum knee flexion angle in patients with knee OA and speculated that the increased soft tissues stiffness may contribute to functional impairment (Ebihara et al., 2020). Although patients with knee OA often experience pathological changes in the muscles around the knee (Rice et al., 2011; Noehren et al., 2018), to the best of our knowledge, no studies have investigated the muscles stiffness in patients with knee OA. Therefore, it is necessary to directly compare the differences in quadriceps stiffness between the healthy subjects and patients with knee OA to obtain a comprehensive understanding of the biomechanical properties of the muscle and to develop an appropriate rehabilitation program for patients with knee OA.

Quantitative assessment of muscle stiffness usually uses biomechanical methods to evaluate changes in the muscletendon length-tension relationship (Rieder et al., 2016). However, it evaluates the stiffness of the entire muscle group and cannot assess changes in the stiffness of individual muscles. A handheld device, MyotonPRO, can measure changes in individual muscle stiffness conveniently and quickly (Chang et al., 2020; Amirova et al., 2021). In our previous studies, we demonstrated that MyotonPRO is a reliable method for assessing the stiffness of the gastrocnemius (Feng et al., 2018) and upper trapezius (Liu CL et al., 2018). Chen et al. (2019) observed good intra- and interrater reliability of MyotonPRO for measuring the stiffness of the superficial quadriceps [vastus lateralis (VL), vastus medialis (VM) and rectus femoris (RF)] (ICC >0.84). Therefore, we compared the differences in the quadriceps stiffness between healthy people and patients with Knee OA using MyotonPRO.

The objectives of this study were to 1) compare the stiffness of the superficial quadriceps (RF, VL, and VM) between healthy individuals and patients with knee osteoarthritis; 2) examine differences of quadriceps stiffness at different angles of the knee joint; and 3) evaluate the correlations between the quadriceps (RF, VL, and VM) stiffness and joint dysfunction in patients with Knee OA.

\section{MATERIALS AND METHODS}

\section{Subjects}

This study had a cross-sectional design. Twenty-five patients with knee OA and 25 healthy, asymptomatic, age-matched subjects were recruited for this study. The sample size was calculated based on a pilot study of 14 subjects. The effect size between the patients with knee OA and healthy controls for VL stiffness was 0.77 . Taking $\alpha$ at $5 \%$ and power at $80 \%$, the estimated sample size was 22 subjects per group. All patients applied to outpatient clinics with knee pain and received an initial clinical assessment (including knee X-rays) to support the clinical diagnosis of knee OA. The patients were included if they met the following criteria (Vaz et al., 2013; Devrimsel et al., 2019): 1) Age greater than 45 years old; 2) clinically diagnosed with knee OA; 3 ) patients diagnosed with grade 2 or 3 knee osteoarthritis according to the criteria proposed by Kellgren and Lawrence; 4) visual analog scale $(\mathrm{VAS})>3$ points in daily activity; and 5) no skin lesions above the measuring regions. Patients' exclusion criteria for this study were based on the following factors: a history of knee or hip or ankle surgery, previous musculoskeletal or joint injuries of lower limb in addition to knee OA, acute inflammation or pain with edema, metabolic diseases, severe deformity of the lower limbs, and BMI $>30 \mathrm{~kg} / \mathrm{m}^{2}$.

\section{Equipment}

In the present study, MyotonPRO (Myoton AS, Tallinn, Estonia) was used to quantify the quadriceps stiffness. The device's basic principles are as follows (Gavronski et al., 2007; Schneider et al., 2015): five short mechanical impulses (the tap interval was $0.8 \mathrm{~s}$ ) were implemented automatically by the device after precompressing the tissue, and these impulses cause mechanical oscillations in the soft tissue being evaluated. Then, MyotonPRO recorded the oscillation information and calculated the soft tissue mechanical parameters. One of these parameters is stiffness (newtons/meter, $\mathrm{N} / \mathrm{m}$ ). The stiffness value was calculated as the maximum acceleration of the oscillation/ maximum displacement of the tissue. The larger the value, the stiffer the tissue. Any data with a coefficient of variation greater than $3 \%$ in any measurement on quintuple scanning mode were remeasured. 
TABLE 1 | The characteristics of the subjects.

\begin{tabular}{|c|c|c|c|}
\hline \multirow[t]{2}{*}{ Women/Men (n) } & $\begin{array}{l}\text { Patients with knee } \\
\text { OA (M } \pm \text { SD) }\end{array}$ & $\begin{array}{c}\text { Healthy } \\
\text { subjects }(M \pm S D)\end{array}$ & $p$ \\
\hline & $17 / 8$ & $17 / 8$ & \\
\hline Age (years) & $62.20 \pm 8.30$ & $59.44 \pm 5.33$ & 0.168 \\
\hline Height (m) & $1.62 \pm 0.06$ & $1.63 \pm 0.06$ & 0.873 \\
\hline Weight (kg) & $63.92 \pm 7.14$ & $62.64 \pm 8.13$ & 0.557 \\
\hline $\mathrm{BMl}\left(\mathrm{kg} / \mathrm{m}^{2}\right)$ & $24.22 \pm 1.96$ & $23.67 \pm 2.67$ & 0.55 \\
\hline WOMAC & $103.48 \pm 27.73$ & - & - \\
\hline
\end{tabular}

WOMAC, the Western Ontario and McMaster Universities Osteoarthritis Index scores.

\section{Procedures}

Demographic information such as age, sex, weight, height, and BMI were recorded before the experiment. The Western Ontario and McMaster Universities Osteoarthritis Index (WOMAC) was used to assess the severity of the symptoms and physical functional disability among the knee OA patients (Salaffi et al., 2003). The questionnaire included 24 questions divided into three subscales: pain, stiffness and physical function. Each question was answered based on a validated 10-point numerical rating scale. The WOMAC has demonstrated acceptable validity and reliability in individuals with Knee OA (Bellamy et al., 1988). The total WOMAC scores were defined as the sum of 24 items, ranging from 0 to 240 .

\section{Measurement of the Quadriceps Stiffness}

The room temperature was maintained at $25^{\circ} \mathrm{C}$ throughout all tests. The quadriceps was subdivided into the rectus femoris $(\mathrm{RF})$, vastus lateralis $(\mathrm{VL})$, vastus medialis $(\mathrm{VM})$, and vastus intermedius (VI). Because the VI is deep inside the RF, the stiffness of the VI cannot be measured by the MyotonPRO. According to previous studies, the measurement region for RF stiffness was two-thirds of the length between the anterior superior iliac spine (ASIS) and the superior pole of the patella (Agyapong-Badu et al., 2016; Chen et al., 2019). The stiffness of the VL and VM was measured at the most salient point of the muscle belly (near the knee joint) (Chen et al., 2019). The measurement sites were marked by the same experienced physical therapist. The symptomatic knees of the patients with knee OA were used for all measurements. When both knees were symptomatic, the most affected knee was evaluated (León-Ballesteros et al., 2020). We measured the stiffness of the soft tissues in the dominant legs in healthy participants. The dominant leg of the subject was determined by which leg they used when they were asked to kick a ball (Lenskjold et al., 2015).

Before the stiffness assessment, the participants were allowed to rest for $5 \mathrm{~min}$. The stiffness measurement was taken with the participant in the sitting position with the knee bent at $60^{\circ}$ and $90^{\circ}$. The knee angle was maintained by an angle-adjustable knee orthosis. The order of stiffness measurements was RF, VL, and VM. The MyotonPRO was applied perpendicular to the surface of the muscle being examined. The subjects were asked to refrain from talking,
TABLE 2| Stiffness of the RF, VL, and VM between the patients with Knee OA and the healthy subjects.

\begin{tabular}{lcccc}
\hline $\begin{array}{l}\text { Knee } \\
\text { flexion }\end{array}$ & & $\begin{array}{c}\text { Patients with knee } \\
\text { OA (M } \pm \text { SD) }\end{array}$ & $\begin{array}{c}\text { Healthy } \\
\text { subjects (M } \pm \text { SD) }\end{array}$ & $\boldsymbol{p}$ \\
\hline $60^{\circ}$ & $\mathrm{RF}$ & $268.68 \pm 37.21$ & $262.96 \pm 31.10$ & 0.558 \\
& $\mathrm{VL}$ & $288.80 \pm 29.78$ & $254.88 \pm 29.33$ & $0.001^{\text {** }}$ \\
& $\mathrm{VM}$ & $260.92 \pm 30.68$ & $246.80 \pm 30.56$ & 0.110 \\
$90^{\circ}$ & $\mathrm{RF}$ & $292.48 \pm 37.86$ & $286.32 \pm 34.42$ & 0.987 \\
& $\mathrm{VL}$ & $321.92 \pm 27.47$ & $289.88 \pm 26.81$ & $0.001^{\text {** }}$ \\
& $\mathrm{VM}$ & $290.36 \pm 39.22$ & $291.40 \pm 40.07$ & 0.926
\end{tabular}

$R F$, rectus femoris; VL, vastus lateralis; $V M$, vastus medialis. ${ }^{* *} \mathrm{p}<0.05$.

to remain relaxed, and to avoid any muscle contraction during the stiffness measurements.

\section{Statistical Analysis}

SPSS Version 22.0 software (IBM, Armonk, NY, United States) was used for the data analysis. The demographic information was calculated by descriptive statistics. The Shapiro-Wilk test was used to assess the normal distribution of the data. Homogeneity of variances was tested using Levene's test. Independent $t$ tests were performed to compare the demographic data between individuals with and without knee OA. Comparisons between RF, VL, and VM stiffness in the healthy subjects and the patients with knee OA were evaluated using an independent sample $t$-test. A paired-sample $t$-test was performed for quadriceps stiffness differences at different knee angles. The correlation between RF, VL, VM stiffness, and WOMAC scores was demonstrated by Pearson correlation analysis ( $\mathrm{r}$ ). All measurement data are expressed as the mean \pm standard deviation, and all $p<0.05$ indicated a significant level.

\section{RESULTS}

\section{Demographic Information}

Demographic information for all participants regarding age, weight, height, and BMI is depicted in Table 1. The clinical assessment report regarding WOMAC is also presented in Table 1. There were no significant differences in age, height, weight, or BMI $(p>0.05)$.

\section{RF, VL, and VM Stiffness Between the Patients With Knee OA and Healthy Subjects}

The mean stiffness values of the RF, VL and VM in the patients with knee OA and healthy controls are summarized in Table 2. The VL stiffness of patients with knee OA was greater than that of the healthy subjects at flexion $60^{\circ}$ of the knee joint $(p<0.05)$. Similarly, compared with the healthy subject group, the patients in the knee OA group had greater VL stiffness at $90^{\circ}$ of knee flexion $(p<0.05)$. However, no between-group differences were observed in the RF and VM stiffness measured at $60^{\circ}$ and $90^{\circ}$ of knee flexion $(p>0.05)$. 


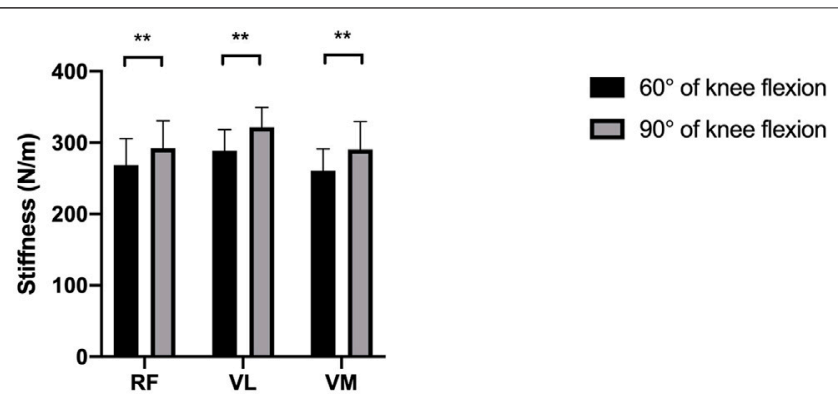

FIGURE 1 | The difference between the mean stiffness of the quadriceps at $60^{\circ}$ and $90^{\circ}$ of knee flexion in patients with knee OA. ${ }^{* *} p<0.05$.

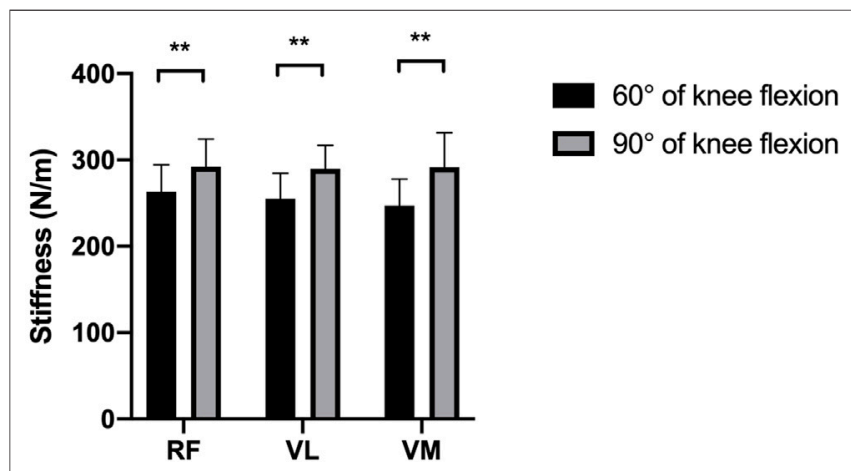

FIGURE 2|The difference between the mean stiffness of the quadriceps at $60^{\circ}$ and $90^{\circ}$ of knee flexion in healthy subjects. ${ }^{*} p<0.05$.

TABLE 3 | Correlation of RF, VL, and VM stiffness and WOMAC scores.

\begin{tabular}{lccc} 
Knee flexion & & \multicolumn{2}{c}{ WOMAC } \\
\cline { 3 - 4 } & & $\boldsymbol{r}$ & $\boldsymbol{p}$ \\
\hline $60^{\circ}$ & $\mathrm{RF}$ & 0.297 & 0.149 \\
& $\mathrm{VL}$ & 0.508 & $0.010^{* \star}$ \\
& $\mathrm{VM}$ & 0.284 & 0.169 \\
$90^{\circ}$ & $\mathrm{RF}$ & 0.283 & 0.170 \\
& $\mathrm{VL}$ & 0.456 & $0.022^{\text {** }}$ \\
& $\mathrm{VM}$ & 0.328 & 0.110
\end{tabular}

$R F$, rectus femoris; $V L$, vastus lateralis; $V M$, vastus medialis; WOMAC, the Western Ontario and McMaster Universities Osteoarthritis Index scores. ${ }^{* *} \mathrm{p}<0.05$.

\section{RF, VL, and VM Stiffness at Different Degrees of Knee Flexion}

Figures 1, 2 reveal the stiffness of RF, VL, and VM at $60^{\circ}$ and $90^{\circ}$ of knee flexion in patients with knee OA and healthy subjects, respectively. A significant difference was found in the RF, VL, and VM stiffness between the $60^{\circ}$ of knee flexion and $90^{\circ}$ flexion of the knee joint in the patients with knee OA $(p<0.05)$. Similarly, in the healthy subject group, there was a significant increase in the $\mathrm{RF}, \mathrm{VL}$, and VM stiffness at $90^{\circ}$ flexion of the knee joint compared with that at $60^{\circ}$ of knee flexion $(p<0.05)$.

\section{The Correlation Between RF, VL, VM Stiffness, and WOMAC Scores}

In patients with Knee OA, Pearson correlation analysis tests were used to indicate the relationship between RF, VL, VM stiffness, and WOMAC scores. As shown in Table 3, there was a significant positive correlation $(r=0.508, p=0.010)$ between the WOMAC scores and the VL stiffness measured at $60^{\circ}$ of knee flexion. At $90^{\circ}$ of knee joint flexion, a significant positive correlation was found between the WOMAC scores and the VL stiffness $(r=0.456, p=$ $0.022)$. However, no significant correlation was apparent between $\mathrm{RF}$ and VM stiffness and WOMAC scores, regardless of knee position $(p>0.05)$. The stiffer the VL is, the greater the level of physical dysfunction in knee OA patients.

\section{DISCUSSION}

This is the first study to compare the stiffness of the VL, RF and VM between subjects with Knee OA and healthy controls. The present study aimed to investigate the difference in the stiffness of the VL, RF and VM between subjects with and without knee OA. The main results of the study demonstrated that increased VL stiffness was found in subjects with knee OA compared to healthy controls. Furthermore, the findings from this study revealed that the quadriceps stiffness increased with knee flexion. More importantly, our study also established a relationship between the stiffness of the VL, RF and VM and the degree of disability among subjects with knee OA. We found a significant positive correlation between the VL stiffness index and WOMAC scores.

We observed the VL stiffness among subjects with Knee OA was higher than that among healthy controls. The VL stiffness was increased by $11.8 \%$ at $60^{\circ}$ of knee flexion and by $10.0 \%$ at $90^{\circ}$ of knee flexion compared to the control group. Although no previous studies have made a direct comparison as in our study, previous studies have reported that a reduction in muscle flexibility may be associated with anterior knee pain (Witvrouw et al., 2000; Piva et al., 2005). A 2-year prospective study revealed that there was significantly less flexibility of the quadriceps in subjects with patellofemoral pain (Witvrouw et al., 2000). They found that the flexibility of the quadriceps in subjects with patellofemoral pain was reduced by $6.1 \%$ compared to the control group. Similarly, Piva et al. (2005) observed that patients with patellofemoral pain syndrome had a reduced knee range of motion. In the study of Witvrouw et al. (2001), significantly lower flexibility of the quadriceps in individuals with patellar tendinitis was obtained as compared with the control group (Witvrouw et al., 2001). A recent study evaluated the stiffness of the RF and VL in athletes with patellar tendinopathy using shear wave elastography, and they observed that the VL stiffness was increased by $26.5 \%$ in athletes with patellar tendinopathy (Zhang et al., 2017). More recently, Zhou et al. (2020) reported that a stiffer medial gastrocnemius but not the lateral gastrocnemius was found in individuals with plantar fasciitis. The knee extensor plays an important role in dissipating kinetic energy during walking, running and landing (Decker et al., 2003), and a decrease in the flexibility of the quadriceps 
muscle might contribute to increasing the loading on the patellofemoral joint (Piva et al., 2005). Some studies have investigated the effects of soft tissue release in patients with knee OA (Tarabichi and Tarabichi, 2010; Atkins and Eichler, 2013). Atkins and Eichler, 2013 conducted a randomized controlled trial and observed a significant difference in the WOMAC score between the self-massage intervention group (applied on the quadriceps) and the control group after intervention. And Field (2016) suggested the massage therapy on quadriceps and hamstrings could reduce pain and increase the knee ROM. Tarabichi and Tarabichi (2010) also found a significant increase in knee ROM after quadriceps release alone in patients with knee OA. The findings from the present study indicate that releasing the quadriceps (especially VL muscle) may have a positive effect on the prevention and treatment of patients with knee OA. Therefore, additional studies need to be conducted to investigate the effectiveness of releasing the tightness of the VL for subjects with knee OA.

As shown in Figure 1 and Figure 2, the stiffness of RF, VL, and $\mathrm{VM}$ in $90^{\circ}$ flexion of the knee joint was significantly higher than that in $60^{\circ}$ flexion of the knee, regardless of whether patients with knee OA or healthy subjects were included. Similarly, Coombes et al. (2018) reported that the stiffness of the quadriceps muscle quantified with shear wave elastography increased with knee flexion. Xu et al. (2018) observed that the passive tension of RF, VL and VM started to increase at $51^{\circ}, 48^{\circ}$, and $45^{\circ}$ flexion, respectively, of the knee joint in rowers (Xu et al., 2018). These cases demonstrate the strainingstiffening behavior of soft tissue after the fibers become tight (DeWall et al., 2014). Chen et al. (2019) measured the stiffness of $\mathrm{RF}, \mathrm{VL}$ and VM in young healthy participants at different angles of knee flexion using MyotonPRO, and they observed an increase of 11.38 and $18.59 \%$ in VL stiffness and VM stiffness in the nondominant limb from $60^{\circ}$ to $90^{\circ}$ flexion of the knee, respectively, while there was no significant difference in RF stiffness. Their results for VL and VM stiffness are similar to our results. In the present study, changing from 60 to $90^{\circ}$ flexion of the knee resulted in 13.73 and $18.07 \%$ VL and VM stiffness in the control group, respectively, but we also found an increase of $8.88 \%$ in RF stiffness. Possible reasons for different variations in RF stiffness measurements may include the different measurement methodologies and the differences in the participants being studied.

In the present study, we also detected a positive correlation between VL stiffness and WOMAC scores in patients with knee OA. To the best of our knowledge, this is the first study to investigate the correlation between quadriceps stiffness and the level of function of patients with knee OA. Some previous studies also focused on the correlations between soft tissue stiffness and pain intensity or the functional level of the patients (Zhang et al., 2014; Koppenhaver et al., 2020; Zhou et al., 2020). In the study of Koppenhaver et al. (2020), lumbar muscle stiffness was greater in individuals with low back pain than in asymptomatic participants and was associated with pain intensity as quantified with the numeric pain rating scale and the level of disability measured by the Oswestry Disability Index (Koppenhaver et al., 2020). Zhou et al. (2020) observed that the stiffness of the medial gastrocnemius was positively correlated with pain intensity in patients with plantar fasciitis. Significant correlations were found between the elastic properties of the patellar tendon and pain and dysfunctions in athletes with patellar tendinopathy (Zhang et al., 2014). The above evidence indicates that soft tissue stiffness may be related to some functional outcomes in patients with musculoskeletal disorders. Chino and Takahashi et al. (2015) and Chino and Takahashi et al. (2016) found that increased muscle stiffness can contribute to an increase in passive joint stiffness. Miyamoto and Hirata (2019) found that muscle stiffness was negatively correlated with joint ROM. Ebihara et al. (2020) investigated the correlation between the passive stiffness of the quadriceps tendon and the gait parameters in the swing phase during gait, and they found that there was a negative correlation between the stiffness of the quadriceps tendon and gait speed and step length in patients with knee OA. Therefore, we speculate that the positive correlation between VL stiffness and WOMAC scores may be caused by the increased VL stiffness leading to decreased knee ROM or increased knee joint stiffness or further increased difficulty in performing activities of daily living in patients with knee OA.

There are some limitations of this study. First, only one point each in the RF, VL and VM was assessed, and our findings could not be generalized to other regions of the quadriceps muscle. Second, although we suggested that subjects be completely relaxed during the stiffness measurements, electromyography was not used to monitor the quadriceps muscle activity and ensure it was not firing. Third, the order of stiffness measurement of quadriceps was not randomized, which may introduce potential bias. In addition, due to the limitations of MyotonPRO, we did not investigate the stiffness of the vastus intermedius. Finally, this study indicated that the VL stiffness was related to the WOMAC score. However, it was not clear whether the VL stiffness affected the level of function in patients with knee OA. A prospective study is necessary to ascertain the cause and effect relationship between passive muscle stiffness and the degree of function in patients with knee OA.

\section{CONCLUSION}

Patients with knee OA have an increase in the stiffness of the vastus lateralis, and the stiffness of the quadriceps is increased with knee flexion in individuals with and without knee OA. In addition, the stiffness of the vastus lateralis is associated with the WOMAC score in patients with knee OA.

\section{DATA AVAILABILITY STATEMENT}

The raw data supporting the conclusion of this article will be made available by the authors, without undue reservation.

\section{ETHICS STATEMENT}

This study was approved by the Human Subjects Ethics Committee of Luoyang Orthopaedic Hospital of Henan Province (KY2020-003-02). Prior to this study, all participants 
provided written informed consent, and the experimental procedure was fully explained to each subject. All of the experimental procedures adhered to the principles of the Declaration of Helsinki.

\section{AUTHOR CONTRIBUTIONS}

T-TC, Y-CZ and FL carried out the experiments and collection data. Z-JZ, J-YG and ZL designed the experiments. Z-JZ, Y-PL and $\mathrm{X}-\mathrm{QW}$ analyzed the experimental results. T-TC and $\mathrm{Y}-\mathrm{CZ}$

\section{REFERENCES}

Agyapong-Badu, S., Warner, M., Samuel, D., and Stokes, M. (2016). Measurement of Ageing Effects on Muscle Tone and Mechanical Properties of Rectus Femoris and Biceps Brachii in Healthy Males and Females Using a Novel Hand-Held Myometric Device. Arch. Gerontol. Geriatr. 62, 59-67. doi:10.1016/ j.archger.2015.09.011

Amirova, L. E., Plehuna, A., Rukavishnikov, I. V., Saveko, A. A., Peipsi, A., and Tomilovskaya, E. S. (2021). Sharp Changes in Muscle Tone in Humans under Simulated Microgravity. Front. Physiol. 12, 661922. doi:10.3389/ fphys.2021.661922

Andrade, R. J., Lacourpaille, L., Freitas, S. R., McNair, P. J., and Nordez, A. (2016). Effects of Hip and Head Position on Ankle Range of Motion, Ankle Passive Torque, and Passive Gastrocnemius Tension. Scand. J. Med. Sci. Sports 26, 41-47. doi:10.1111/sms.12406

Atkins, D. V., and Eichler, D. A. (2013). The Effects of Self-Massage on Osteoarthritis of the Knee: A Randomized, Controlled Trial. Int. J. Ther. Massage Bodyw. 6, 4-14. doi:10.3822/ijtmb.v6i1.119

Avrillon, S., Hug, F., and Guilhem, G. (2018). Between-muscle Differences in Coactivation Assessed Using Elastography. J. Electromyogr. Kinesiol. 43, 88-94. doi:10.1016/j.jelekin.2018.09.007

Bellamy, N., Buchanan, W. W., Goldsmith, C. H., Campbell, J., and Stitt, L. W. (1988). Validation Study of WOMAC: a Health Status Instrument for Measuring Clinically Important Patient Relevant Outcomes to Antirheumatic Drug Therapy in Patients with Osteoarthritis of the Hip or Knee. J. Rheumatol. 15, 1833-1840.

Breda, S. J., van der Vlist, A., de Vos, R.-J., Krestin, G. P., and Oei, E. H. G. (2020). The Association between Patellar Tendon Stiffness Measured with Shear-Wave Elastography and Patellar Tendinopathy-A Case-Control Study. Eur. Radiol. 30, 5942-5951. doi:10.1007/s00330-020-06952-0

Chang, T.-T., Li, Z., Wang, X.-Q., and Zhang, Z.-J. (2020). Stiffness of the Gastrocnemius-Achilles Tendon Complex between Amateur Basketball Players and the Non-athletic General Population. Front. Physiol. 11, 606706. doi:10.3389/fphys.2020.606706

Chen, G., Wu, J., Chen, G., Lu, Y., Ren, W., Xu, W., et al. (2019). Reliability of a Portable Device for Quantifying Tone and Stiffness of Quadriceps Femoris and Patellar Tendon at Different Knee Flexion Angles. PloS one 14, e0220521. doi:10.1371/journal.pone.0220521

Chino, K., and Takahashi, H. (2015). The Association of Muscle and Tendon Elasticity with Passive Joint Stiffness: In Vivo Measurements Using Ultrasound Shear Wave Elastography. Clin. Biomech. 30, 1230-1235. doi:10.1016/ j.clinbiomech.2015.07.014

Chino, K., and Takahashi, H. (2016). Measurement of Gastrocnemius Muscle Elasticity by Shear Wave Elastography: Association with Passive Ankle Joint Stiffness and Sex Differences. Eur. J. Appl. Physiol. 116, 823-830. doi:10.1007/ s00421-016-3339-5

Cho, H.-y., Kim, E.-H., Kim, J., and Yoon, Y. W. (2015). Kinesio Taping Improves Pain, Range of Motion, and Proprioception in Older Patients with Knee Osteoarthritis. Am. J. Phys. Med. Rehabil. 94, 192-200. doi:10.1097/ PHM.0000000000000148

Coombes, B. K., Ziegenfuss, B., David, M., Badya, R., van den Hoorn, W., Hug, F., et al. (2018). Heterogeneity of Passive Elastic Properties within the Quadriceps drafted the manuscript. All authors reviewed and approved the submission of the manuscript.

\section{FUNDING}

This study were supported by Project of Science Research of Traditional Chinese Medicine of Henan Province of China (2019ZY1028) and key project of decision-making consultation research of Shanghai Municipal People's government (2021A-047).

Femoris Muscle-Tendon Unit. Eur. J. Appl. Physiol. 118, 213-221. doi:10.1007/ s00421-017-3763-1

Cross, M., Smith, E., Hoy, D., Nolte, S., Ackerman, I., Fransen, M., et al. (2014). The Global burden of Hip and Knee Osteoarthritis: Estimates from the Global burden of Disease 2010 Study. Ann. Rheum. Dis. 73, 1323-1330. doi:10.1136/ annrheumdis-2013-204763

Davis, H. C., Luc-Harkey, B. A., Seeley, M. K., Troy Blackburn, J., and Pietrosimone, B. (2019). Sagittal Plane Walking Biomechanics in Individuals with Knee Osteoarthritis after Quadriceps Strengthening. Osteoarthr. Cartil. 27, 771-780. doi:10.1016/j.joca.2018.12.026

Decker, M. J., Torry, M. R., Wyland, D. J., Sterett, W. I., and Richard Steadman, J. (2003). Gender Differences in Lower Extremity Kinematics, Kinetics and Energy Absorption during landing. Clin. Biomech. 18, 662-669. doi:10.1016/ s0268-0033(03)00090-1

Devrimsel, G., Metin, Y., and Serdaroglu Beyazal, M. (2019). Short-term Effects of Neuromuscular Electrical Stimulation and Ultrasound Therapies on Muscle Architecture and Functional Capacity in Knee Osteoarthritis: a Randomized Study. Clin. Rehabil. 33, 418-427. doi:10.1177/0269215518817807

DeWall, R. J., Slane, L. C., Lee, K. S., and Thelen, D. G. (2014). Spatial Variations in Achilles Tendon Shear Wave Speed. J. Biomech. 47, 2685-2692. doi:10.1016/ j.jbiomech.2014.05.008

Dillon, C. F., Rasch, E. K., Gu, Q., and Hirsch, R. (2006). Prevalence of Knee Osteoarthritis in the United States: Arthritis Data from the Third National Health and Nutrition Examination Survey 1991-94. J. Rheumatol. 33, 2271-2279.

Du, C., Smith, A., Avalos, M., South, S., Crabtree, K., Wang, W., et al. (2019). Blueberries Improve Pain, Gait Performance, and Inflammation in Individuals with Symptomatic Knee Osteoarthritis. Nutrients 11, 290. doi:10.3390/ nu1 1020290

Ebihara, B., Fukaya, T., and Mutsuzaki, H. (2020). Relationship between Quadriceps Tendon Young's Modulus and Maximum Knee Flexion Angle in the Swing Phase of Gait in Patients with Severe Knee Osteoarthritis. Medicina 56, 437. doi:10.3390/medicina56090437

Feng, Y. N., Li, Y. P., Liu, C. L., and Zhang, Z. J. (2018). Assessing the Elastic Properties of Skeletal Muscle and Tendon Using Shearwave Ultrasound Elastography and MyotonPRO. Sci. Rep. 8, 17064. doi:10.1038/s41598-01834719-7

Field, T. (2016). Knee Osteoarthritis Pain in the Elderly Can Be Reduced by Massage Therapy, Yoga and Tai Chi: A Review. Complement. Therapies Clin. Pract. 22, 87-92. doi:10.1016/j.ctcp.2016.01.001

Gavronski, G., Veraksitš, A., Vasar, E., and Maaroos, J. (2007). Evaluation of Viscoelastic Parameters of the Skeletal Muscles in Junior Triathletes. Physiol. Meas. 28, 625-637. doi:10.1088/0967-3334/28/6/002

Geertsen, S. S., Kirk, H., Lorentzen, J., Jorsal, M., Johansson, C. B., and Nielsen, J. B. (2015). Impaired Gait Function in Adults with Cerebral Palsy Is Associated with Reduced Rapid Force Generation and Increased Passive Stiffness. Clin. Neurophysiol. 126, 2320-2329. doi:10.1016/j.clinph.2015.02.005

Hirata, K., Miyamoto-Mikami, E., Kanehisa, H., and Miyamoto, N. (2016). Musclespecific Acute Changes in Passive Stiffness of Human Triceps Surae after Stretching. Eur. J. Appl. Physiol. 116, 911-918. doi:10.1007/s00421-016-3349-3

Hunter, D. J., and Bierma-Zeinstra, S. (2019). Osteoarthritis. Lancet 393, 1745-1759. doi:10.1016/S0140-6736(19)30417-9 
Ishikawa, H., Muraki, T., Morise, S., Sekiguchi, Y., Yamamoto, N., Itoi, E., et al. (2017). Changes in Stiffness of the Dorsal Scapular Muscles before and after Computer Work: a Comparison between Individuals with and without Neck and Shoulder Complaints. Eur. J. Appl. Physiol. 117, 179-187. doi:10.1007/ s00421-016-3510-z

Koppenhaver, S., Gaffney, E., Oates, A., Eberle, L., Young, B., Hebert, J., et al. (2020). Lumbar Muscle Stiffness Is Different in Individuals with Low Back Pain Than Asymptomatic Controls and Is Associated with Pain and Disability, but Not Common Physical Examination Findings. Musculoskelet. Sci. Pract. 45, 102078. doi:10.1016/j.msksp.2019.102078

Lenskjold, A., Kongsgaard, M., Larsen, J. O., Nielsen, R. H., Kovanen, V., Aagaard, P., et al. (2015). The Influence of Physical Activity during Youth on Structural and Functional Properties of the Achilles Tendon. Scand. J. Med. Sci. Sports 25, 25-31. doi:10.1111/sms.12143

León-Ballesteros, S., Espinosa-Morales, R., Clark-Peralta, P., Gómez-Pineda, A. G., and Guadarrama-Becerril, J. H. (2020). Kinesiotape and Quadriceps Strengthening with Elastic Band in Women with Knee Osteoarthritis and Overweight or Obesity. A Randomized Clinical Trial. Reumatol. Clín. 16, 11-16. doi:10.1016/j.reuma.2018.03.001

Liu CL, C. L., Feng, Y. N., Zhang, H. Q., Li, Y. P., Zhu, Y., and Zhang, Z. J. (2018). Assessing the Viscoelastic Properties of Upper Trapezius Muscle: Intra- and Inter-tester Reliability and the Effect of Shoulder Elevation. J. Electromyogr. Kinesiol. 43, 226-229. doi:10.1016/j.jelekin.2017.09.007

Liu Q, Q., Wang, S., Lin, J., and Zhang, Y. (2018). The burden for Knee Osteoarthritis Among Chinese Elderly: Estimates from a Nationally Representative Study. Osteoarthr. Cartil. 26, 1636-1642. doi:10.1016/ j.joca.2018.07.019

Lu, Z., Li, X., Chen, R., and Guo, C. (2018). Kinesio Taping Improves Pain and Function in Patients with Knee Osteoarthritis: A Meta-Analysis of Randomized Controlled Trials. Int. J. Surg. 59, 27-35. doi:10.1016/j.ijsu.2018.09.015

Michael, J. W.-P., Schlüter-Brust, K. U., and Eysel, P. (2010). The Epidemiology, Etiology, Diagnosis, and Treatment of Osteoarthritis of the Knee. Deutsches Arzteblatt Int. 107, 152-162. doi:10.3238/arztebl.2010.0152

Miyamoto, N., and Hirata, K. (2019). Moderate Associations of Muscle Elasticity of the Hamstring with Hip Joint Flexibility. Int. J. Sports Med. 40, 717-724. doi:10.1055/a-0981-7282

Noehren, B., Kosmac, K., Walton, R. G., Murach, K. A., Lyles, M. F., Loeser, R. F., et al. (2018). Alterations in Quadriceps Muscle Cellular and Molecular Properties in Adults with Moderate Knee Osteoarthritis. Osteoarthr. Cartil. 26, 1359-1368. doi:10.1016/j.joca.2018.05.011

Nordez, A., Gross, R., Andrade, R., Le Sant, G., Freitas, S., Ellis, R., et al. (2017). Non-Muscular Structures Can Limit the Maximal Joint Range of Motion during Stretching. Sports Med. 47, 1925-1929. doi:10.1007/s40279-017-0703-5

Perlman, A., Fogerite, S. G., Glass, O., Bechard, E., Ali, A., Njike, V. Y., et al. (2019). Efficacy and Safety of Massage for Osteoarthritis of the Knee: a Randomized Clinical Trial. J. Gen. Intern. Med. 34, 379-386. doi:10.1007/s11606-018-4763-5

Piva, S. R., Goodnite, E. A., and Childs, J. D. (2005). Strength Around the Hip and Flexibility of Soft Tissues in Individuals with and without Patellofemoral Pain Syndrome. J. Orthop. Sports Phys. Ther. 35, 793-801. doi:10.2519/jospt.2005.35.12.793

Rice, D. A., McNair, P. J., and Lewis, G. N. (2011). Mechanisms of Quadriceps Muscle Weakness in Knee Joint Osteoarthritis: the Effects of Prolonged Vibration on Torque and Muscle Activation in Osteoarthritic and Healthy Control Subjects. Arthritis Res. Ther. 13, R151. doi:10.1186/ar3467

Rieder, F., Wiesinger, H.-P., Kösters, A., Müller, E., and Seynnes, O. R. (2016). Whole-body Vibration Training Induces Hypertrophy of the Human Patellar Tendon. Scand. J. Med. Sci. Sports 26, 902-910. doi:10.1111/sms.12522

Safiri, S., Kolahi, A.-A., Smith, E., Hill, C., Bettampadi, D., Mansournia, M. A., et al. (2020). Global, Regional and National burden of Osteoarthritis 1990-2017: a Systematic Analysis of the Global Burden of Disease Study 2017. Ann. Rheum. Dis. 79, 819. doi:10.1136/annrheumdis-2019-216515

Salaffi, F., Leardini, G., Canesi, B., Mannoni, A., Fioravanti, A., Caporali, R., et al. (2003). Reliability and Validity of the Western Ontario and McMaster Universities (WOMAC) Osteoarthritis Index in Italian Patients with
Osteoarthritis of the Knee. Osteoarthr. Cartil. 11, 551-560. doi:10.1016/ s1063-4584(03)00089-x

Schneider, S., Peipsi, A., Stokes, M., Knicker, A., and Abeln, V. (2015). Feasibility of Monitoring Muscle Health in Microgravity Environments Using Myoton Technology. Med. Biol. Eng. Comput. 53, 57-66. doi:10.1007/s11517-0141211-5

Simic, M., Harmer, A. R., Agaliotis, M., Nairn, L., Bridgett, L., March, L., et al. (2021). Clinical Risk Factors Associated with Radiographic Osteoarthritis Progression Among People with Knee Pain: a Longitudinal Study. Arthritis Res. Ther. 23 (1), 160. doi:10.1186/s13075-021-02540-9

Szilagyi, I. A., Waarsing, J. H., Schiphof, D., van Meurs, J. B. J., and BiermaZeinstra, S. M. A. (2021). Towards Sex-specific Osteoarthritis Risk Models: Evaluation of Risk Factors for Knee Osteoarthritis in Males and Females. Rheumatology (Oxford), keab378. doi:10.1093/rheumatology/keab378

Tarabichi, S., and Tarabichi, Y. (2010). Can an Anterior Quadriceps Release Improve Range of Motion in the Stiff Arthritic Knee? J. Arthroplasty 25, 571-575. doi:10.1016/j.arth.2009.04.015

Vaz, M. A., Baroni, B. M., Geremia, J. M., Lanferdini, F. J., Mayer, A., Arampatzis, A., et al. (2013). Neuromuscular Electrical Stimulation (NMES) Reduces Structural and Functional Losses of Quadriceps Muscle and Improves Health Status in Patients with Knee Osteoarthritis. J. Orthop. Res. 31, 511-516. doi:10.1002/jor.22264

Witvrouw, E., Lysens, R., Bellemans, J., Cambier, D., and Vanderstraeten, G. (2000). Intrinsic Risk Factors for the Development of Anterior Knee Pain in an Athletic Population: A Two-Year Prospective Study. Am. J. Sports Med. 28, 480-489. doi:10.1177/03635465000280040701

Witvrouw, E., Bellemans, J., Lysens, R., Danneels, L., and Cambier, D. (2001). Intrinsic Risk Factors for the Development of Patellar Tendinitis in an Athletic Population. Am. J. Sports Med. 29, 190-195. doi:10.1177/ 03635465010290021201

Xu, J., Hug, F., and Fu, S. N. (2018). Stiffness of Individual Quadriceps Muscle Assessed Using Ultrasound Shear Wave Elastography during Passive Stretching. J. Sport Health Sci. 7, 245-249. doi:10.1016/j.jshs.2016.07.001

Zhang, Z. J., Ng, G. Y.-f., Lee, W. C., and Fu, S. N. (2014). Changes in Morphological and Elastic Properties of Patellar Tendon in Athletes with Unilateral Patellar Tendinopathy and Their Relationships with Pain and Functional Disability. PloS one 9, e108337. doi:10.1371/journal.pone.0108337

Zhang, Z. J., Ng, G. Y. F., Lee, W. C., and Fu, S. N. (2017). Increase in Passive Muscle Tension of the Quadriceps Muscle Heads in Jumping Athletes with Patellar Tendinopathy. Scand. J. Med. Sci. Sports 27, 1099-1104. doi:10.1111/ sms.12749

Zhou, J.-P., Yu, J.-F., Feng, Y.-N., Liu, C.-L., Su, P., Shen, S.-H., et al. (2020). Modulation in the Elastic Properties of Gastrocnemius Muscle Heads in Individuals with Plantar Fasciitis and its Relationship with Pain. Sci. Rep. 10, 2770. doi:10.1038/s41598-020-59715-8

Conflict of Interest: The authors declare that the research was conducted in the absence of any commercial or financial relationships that could be construed as a potential conflict of interest.

Publisher's Note: All claims expressed in this article are solely those of the authors and do not necessarily represent those of their affiliated organizations, or those of the publisher, the editors and the reviewers. Any product that may be evaluated in this article, or claim that may be made by its manufacturer, is not guaranteed or endorsed by the publisher.

Copyright (C) 2022 Chang, Zhu, Li, Li, Li, Guo, Wang and Zhang. This is an openaccess article distributed under the terms of the Creative Commons Attribution License (CC BY). The use, distribution or reproduction in other forums is permitted, provided the original author(s) and the copyright owner(s) are credited and that the original publication in this journal is cited, in accordance with accepted academic practice. No use, distribution or reproduction is permitted which does not comply with these terms. 